\title{
SPATIAL AND TEMPORAL INTER-RELATIONSHIPS BETWEEN ANOMALIES OF TEMPERATURE, MOISTURE, CLOUD COVER, AND OLR AS OBSERVED BY AIRS/AMSU ON AQUA
}

\author{
Joel Susskind and Gyula Molnar
}

NASA GSFC and GEST/UMBC

\begin{abstract}
AIRS/AMSU is the advanced IR/MW atmospheric sounding system launched on EOS Aqua in May 2002. Products derived from AIRS/AMSU include surface skin temperature and atmospheric temperature profiles; atmospheric humidity profiles, \% cloud cover and cloud top pressure, and OLR. Near real time products, starting with September 2002, have been derived from AIRS/AMSU using the AIRS Science Team Version 5 retrieval algorithm. Results in this paper included products through April 2008. The time period studied is marked by a substantial warming trend of Northern Hemisphere Extropical land surface skin temperatures, as well as pronounced El Niño - La Niña episodes. These both influence the spatial and temporal anomaly patterns of atmospheric temperature and moisture profiles, as well as of cloud cover and Clear Sky and All Sky OLR. The relationships between temporal and spatial anomalies of these parameters over this time period, as determined from AIRS/AMSU observations, are shown below, with particular emphasis on which contribute significantly to OLR anomalies in each of the tropics and extra-tropics. The ability to match this data represents a good test of a model's response to El Niño.
\end{abstract}

Index Terms - Climate data, Anomalies, El Niño, AIRS/AMSU, OLR

\section{THE VERSION 5 AIRS/AMSU DATA SET}

The Goddard DAAC has analyzed AIRS/AMSU data, using the AIRS Science Team Version 5 algorithm, for the period September 2002 to the present (April 2008). The AIRS/AMSU sounding retrieval methodology allows for the retrieval of key atmospheric/surface parameters under partially cloudy conditions. This allows for successful retrievals in up to $80-90 \%$ cloud cover. The AIRS/AMSU Version 5.0 retrieval methodology is essentially a physically-based system. It is independent of GCM except for surface pressure, which is used only in the radiative transfer equation. Cloud parameters and OLR are derived under all cloud conditions.

A data set of accurate, long-term satellite sounder measurements of atmospheric temperature and moisture profiles, cloud cover, and longwave radiative fluxes can help make general circulation models [GCMs] a much more reliable tool of weather and climate change prediction in (at least) two ways: they could provide input for the necessary parameterizations on one hand, and test/validate the model results on the other.

In this paper, we demonstrate the reasonableness and compatibility of DAAC AIRS Version $5.01^{\circ} \times 1^{\circ}$ gridded monthly mean average (Level 3 ) products covering the first $52 / 3$ years of operation covering the period from September 2002 till April 2008. These data are available through the WEB-site of the DAAC at NASA GSFC

(http://disc.gsfc.nasa.gov/data/datapool/AIRS/index.html) in which Version 5.0 products are referred to as "V005". The AIRS data set has a good possibility of extending eventually for a 15 year period.

\section{APPROACH}

Our main aim here is to assess eventual utility of AIRS data for climate research. Here we focus on the consistency of the $52 / 3$ year long series of monthly climatic anomalies, relative to the mean monthly climatology obtained from the averaging of the first five years of each respective AIRS month. Before analyzing the data, all DAAC 5.0 monthly mean fields have been spatially interpolated to fill-in missing data grid points (which were very few, well below $0.1 \%$ of the total number of gridpoints). In addition, elevated terrain is excluded at the appropriate pressure levels. After the AIRS five-year $1^{\circ} \times 1^{\circ}$ monthly mean "climatologies" were generated, we've computed the monthly mean anomalies as area weighted (when areas were larger than the $1^{\circ} \times 1^{\circ}$ grid) differences from the area weighted monthly climatologies. In addition to creating the time series of global, tropical and regional anomalies, we computed linear trends fitted to the anomaly time series of each grid-point. Using these, we generate area averaged anomaly time series and their trends.

\section{RESULTS}

Fig. 1 shows the gridpoint by gridpoint anomaly trends for several important climate parameters: a) surface skin temperature $(\mathrm{K} / \mathrm{yr})$, b) $500 \mathrm{mb}$ specific humidity (\%/year); c) OLR $\left(\mathrm{W} / \mathrm{m}^{2} / \mathrm{yr}\right)$; and d) clear sky OLR (W/m²/yr). Pronounced regional trend patterns are obvious in all the 
fields. Two very significant features are found in Figure 1a. The equatorial Pacific shows a pronounced cooling trend, which is a result of ongoing El Niño/La Niña cycles, as will be shown in Fig. 2. It is interesting to note that tropical land surface skin temperature has cooled during this time period as well. There is also a substantial warming of most northern hemisphere extra-tropical land during this time period. Globally, the area weighted surface skin temperature cooled by $0.02 \mathrm{~K} / \mathrm{y}$ over this period. It should be emphasized that this represents what happened over this time period, and cannot be used to predict future trends, nor is a "global trend" in any way indicative of what is happening over the whole globe.

Several parameters show strong inter-correlations of the spatial trend patterns. Trends of the specific humidity at 500 $\mathrm{mb}$ have a very pronounced spatial structure, especially in the tropics between $120^{\circ} \mathrm{W}$ and $120^{\circ} \mathrm{E}$, that are in phase with the effects of the El Niño - La Niña oscillation. The equatorial drying trend in the vicinity of the dateline is accompanied by a strong moistening trend west of South America that is not directly related to any trends in the surface skin temperature.

Table 1 contains the spatial correlation of the anomaly trends of 6 geophysical parameters highly relevant to global change, including $\mathrm{T}_{\text {skin }}$ and $\mathrm{PCSH}_{500}$. Tropical spatial correlations $\left(23^{\circ} \mathrm{N}-23^{\circ} \mathrm{S}\right)$ are shown in bold, and extratropical spatial correlations (poleward of $40^{\circ}$ ) are shown in normal type. Extratropical trends of $\mathrm{T}_{\text {skin }}$ and $\mathrm{PCSH}_{500}$ show a spatial correlation of 0.58 , while tropical trends have a spatial correlation of only 0.27 , because decreased moisture in the La Niña area is accompanied by a significant increase in moisture in an adjacent area.

Figures $1 \mathrm{c}$ and 1d show anomaly trends of two other geophysical parameters often used to assess climate change, OLR and Clear Sky OLR (OLR $\left.R_{C L R}\right)$. Both OLR and OLR $_{\text {CLR }}$ represent fluxes to space which are computed via radiative transfer based on the retrieved AIRS geophysical parameters. OLR is the computed flux in the presence of the retrieved clouds, and OLR $\mathrm{CLR}_{\text {in }}$ is the flux computed otherwise the same, but assuming no clouds in the field of view (the scene need not be clear). According to theory, OLR $_{C L R}$ increases with increasing surface and atmospheric temperature, and decreases with increasing atmospheric water vapor, especially for moist (tropical) cases. OLR changes are influenced in the same way by changes in temperature and moisture. The single largest factor influencing OLR, and OLR changes, is changes in cloud amount, especially for high cold colds. OLR $\mathrm{CLR}_{\mathrm{R}}$ is not directly affected by cloud cover at all.

Spatial trends of OLR and OLR $\mathrm{CLR}_{\mathrm{R}}$ are very similar to each other, but the magnitude of OLR trends in the tropics is roughly 3 times that of OLR ${ }_{C L R}$. Tropical trends of OLR $R_{C L R}$ are highly anti-correlated with those of $500 \mathrm{mb}$ specific humidity, which is the dominant factor affecting tropical OLR $_{\text {CLR }}$. It is apparent then that roughly $1 / 3$ of the tropical trends of OLR results from changes in atmospheric water vapor, and the remaining $2 / 3$ comes from changes in cloud fraction $(\alpha)$ and cloud top pressure, neither of which are shown in Figure 1. In the extra-tropics, spatial trends in both OLR and OLR ${ }_{C L R}$ are highly correlated with those of $\mathrm{T}_{\text {skin }}$ as expected, because the effects of water vapor on OLR are smaller in dryer cases.

Table 1 includes tropical and extra-tropical spatial trend correlations of all the geophysical parameters discussed thus far, as well as those of $500 \mathrm{mb}$ temperature $\mathrm{T}_{500}$, and \% cloud fraction $\alpha$. In the extra-tropics, spatial trends of atmospheric temperature and water vapor correlate well with those of skin temperature, but those of cloud cover do not correlate well with any of the other geophysical parameters. In the tropics on the other hand, spatial trends of cloud fraction correlate very highly with those of midtropospheric water vapor, but none of the spatial trends of geophysical parameters correlate well with those of surface skin temperature.

Figures $2 \mathrm{a}-2 \mathrm{~d}$ show monthly mean anomalies, averaged from $5^{\circ} \mathrm{N}$ to $5^{\circ} \mathrm{S}$, for every $1^{\circ}$ longitude grid box as a function of time. The vertical lines indicate ocean/land boundaries. The region from $180^{\circ} \mathrm{W}$ to $100^{\circ} \mathrm{W}$ is shown twice for spatial contiguity. The year marker corresponds with January of that year. Figure $2 \mathrm{a}$ shows clearly the oscillation between an EL Niño period in 2002, and La Niña periods, especially at the end of 2007. Tropical spatial trends of $\mathrm{T}_{\text {skin }}$ are generally considerably smaller outside the El Niño region, which goes from roughly $160^{\circ} \mathrm{E}$ to $90^{\circ} \mathrm{W}$, with the exception of Eastern Africa. This region shows some out of phase relationship with the eastern tropical Pacific, as well as with the tropical Indian Ocean.

Figure $2 \mathrm{~b}$ shows that trends in mid-tropospheric specific humidity closely match those of $\mathrm{T}_{\text {skin }}$ in the El Niño /La Niña area, but exhibit some strong out of phase behavior off the west coast of South America. There is a clear eastward propagation of the drying trend over the Atlantic Ocean and Africa, occurring in 2005, toward Indonesia in 2006, consistent with the propagation of cooling trends of SST's during this time period. Figures $2 \mathrm{c}$ and $2 \mathrm{~d}$ show analogous trend time series of OLR and OLR $\mathrm{CLR}_{\text {. The anomaly }}$ patterns correlate very well with each other. This may be at first glance surprising because tropical OLR is influenced to first order by cloud cover, while OLR $_{\text {CLR }}$ is not influenced by cloud cover at all. The key to this is the high correlation between temporal anomalies in cloud cover and those of atmospheric water vapor, which is the main factor influencing tropical clear sky OLR.

Table II shows the temporal anomaly correlations of the same geophysical parameters given in Table 1. Values are given below the diagonal, to be in the same locations as the tropical spatial correlations shown in Table 1. In general, the spatial correlations of tropical temporal anomalies of different geophysical parameters are similar to, and somewhat larger than, those of their trends. 

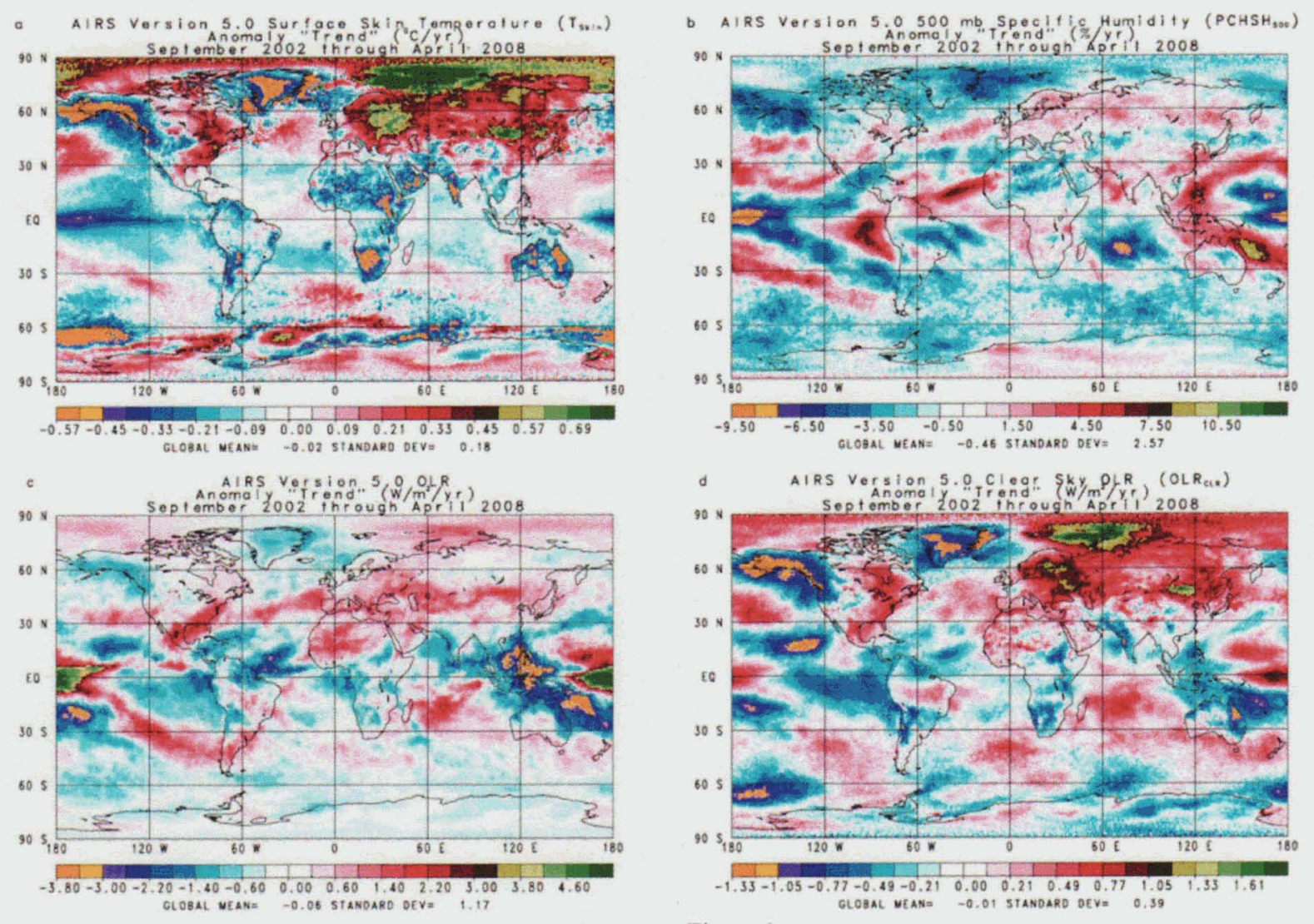

Figure 1

Table 1: Correlations between the $\mathbf{6 8}$ month anomaly trends of selected climatic variables for the tropical $\left(23^{\circ} \mathrm{N}-23^{\circ} \mathrm{S}\right.$ - bold typeface) and for the poleward of $40^{\circ}$ (regular typeface) regions of the trend-maps.

\begin{tabular}{ccccccc}
\hline & $\mathbf{T}_{\text {skin }}$ & $\mathbf{T}_{\mathbf{5 0 0}}$ & PCSH $_{\mathbf{5 0 0}}$ & OLR $_{\text {CLR }}$ & OLR & $\boldsymbol{\alpha}$ \\
\hline $\mathbf{T}_{\text {skin }}$ & --- & 0.63 & 0.58 & 0.90 & 0.68 & 0.15 \\
$\mathbf{T}_{\mathbf{5 0 0}}$ & $\mathbf{0 . 1 7}$ & --- & 0.82 & 0.79 & 0.63 & 0.02 \\
PCSH $_{\mathbf{5 0 0}}$ & $\mathbf{0 . 2 7}$ & $\mathbf{0 . 1 4}$ & --- & 0.67 & 0.50 & 0.05 \\
OLR $_{\text {CLR }}$ & $\mathbf{0 . 0 9}$ & $\mathbf{0 . 3 0}$ & $\mathbf{- 0 . 7 2}$ & --- & 0.75 & 0.11 \\
OLR & $\mathbf{- 0 . 2 7}$ & $\mathbf{- 0 . 1 3}$ & $\mathbf{- 0 . 7 7}$ & $\mathbf{0 . 7 4}$ & -- & -0.38 \\
$\boldsymbol{\alpha}$ & $\mathbf{0 . 1 5}$ & $\mathbf{0 . 1 2}$ & $\mathbf{0 . 6 1}$ & $\mathbf{- 0 . 6 3}$ & $\mathbf{- 0 . 8 6}$ & -- \\
\hline
\end{tabular}

Table 2: Correlations between the AIRS anomaly timeseries of selected climatic variables depicted in the equatorial $\left(5^{\circ} \mathrm{N}-5^{\circ} \mathrm{S}\right)$ Hovmoller Diagrams for the first 68 months of data.

\begin{tabular}{|c|c|c|c|c|c|c|}
\hline & $T_{\text {skin }}$ & $T_{500}$ & $\mathrm{PCSH}_{500}$ & $\overline{\text { OLR }_{\text {CLR }}}$ & OLR & $\alpha$ \\
\hline$\overline{T_{\text {skin }}}$ & $\overline{---}$ & & & & & \\
\hline $\mathbf{T}_{500}$ & 0.61 & --- & & & & \\
\hline $\mathrm{PCSH}_{500}$ & 0.45 & 0.27 & --- & & & \\
\hline OLR $_{\text {CLR }}$ & -0.01 & 0.11 & -0.76 & --- & & \\
\hline OLR & -0.40 & -0.20 & -0.76 & 0.76 & -- & \\
\hline$\alpha$ & 0.22 & 0.12 & 0.66 & -0.75 & -0.92 & -- \\
\hline
\end{tabular}



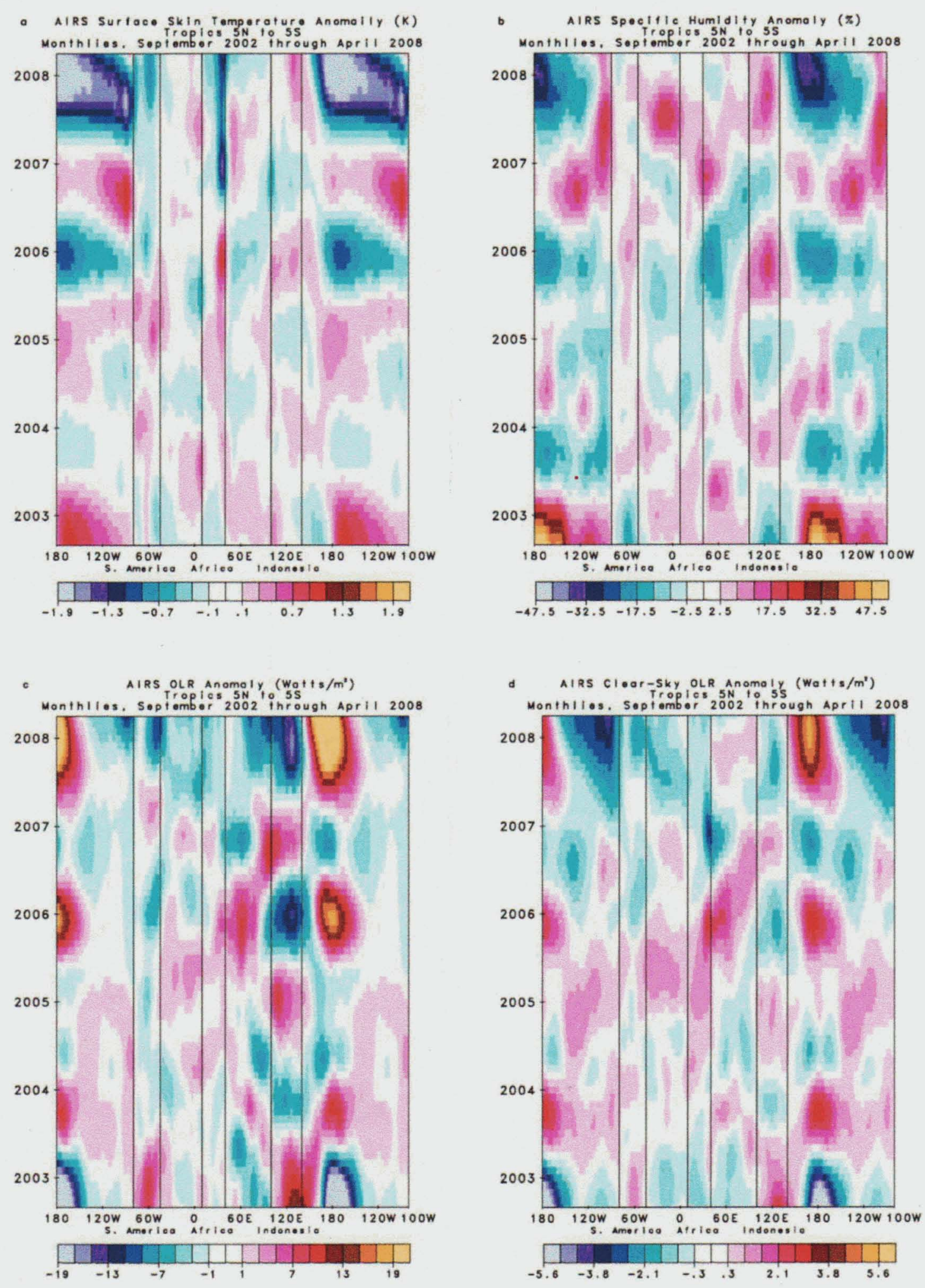

Figure 2 